\title{
25 Research Suare

\section{Silencing NDC80, RAD21 and BUB1B ameliorates intervertebral disc degeneration by promoting proliferation and inhibiting apoptosis of nucleus pulposus cells}

\section{Bin Zhang}

Tianjin Medical University General Hospital

\section{Yueyan Guan}

Tianjin Medical University General Hospital

Weixiao Liu

Tianjin Medical University General Hospital

Wei Guo

Tianjin Medical University General Hospital

\section{Peng Peng}

Tianjin Medical University General Hospital

\section{Cong Xing}

Tianjin Medical University General Hospital

Jingyuan Huang

Tianjin Medical University General Hospital

Chao Sun

Tianjin Medical University General Hospital

\section{Wendong Ruan}

Tianjin Medical University General Hospital

\section{Guangzhi Ning}

Tianjin Medical University General Hospital

\section{Xiaohong Kong}

Nankai University School of Medicine

Shi-Qing Feng ( $\nabla$ sqfeng@tmu.edu.cn )

Tianjin Medical University General Hospital

\section{Research article}

Keywords: Gene silencing, Intervertebral disc degeneration, Nucleus pulposus, Cell cycle, Cell proliferation, Cell apoptosis 
Posted Date: June 19th, 2020

DOl: https://doi.org/10.21203/rs.3.rs-31592/v1

License: (c) (i) This work is licensed under a Creative Commons Attribution 4.0 International License. Read Full License 


\section{Abstract \\ Background}

Intervertebral disc degeneration (IVDD) is a commonly occurring musculoskeletal disorder, which is closely associated with low back pain. Accumulating evidence has demonstrated that dysregulated genes expression profiles play important roles in pathogenesis of IVDD. Hence, the current study was aimed to identify key genes to understand underlying mechanisms and therapeutic targets of IVDD.

\section{Methods}

Microarray datasets of GSE34095, GSE63492 and GSE45856 were downloaded to identify the hub genes that participate in the IVDD pathogenesis. After establishment of rat IVDD models, the expressions of NDC80, BUB1B and RAD21 in rat IVDD samples were evaluated by reverse transcription quantitative PCR (RT-qPCR) and immunochemistry. Subsequently, we assessed the proliferation, cycle and apoptosis of nucleus pulposus (NP) cells that transfected with siRNA-NDC80, siRNA-BUB1B and siRNA-RAD21.

\section{Results}

Our results showed indicated that NDC80, BUB1B and RAD21 were the key pathogenic genes with higher expression in IVDD rats, and silencing of NDC80, BUB1B and RAD21 gene could promote the aggrecan and collagen II synthesis, cell cycle and proliferation of NP cells, and inhibit NP cells apoptosis.

\section{Conclusion}

Our study suggests that silencing NDC80, RAD21 and BUB1B genes ameliorates intervertebral disc degeneration by promoting proliferation and inhibiting apoptosis of nucleus pulposus cells.

\section{Background}

Intervertebral disc degeneration (IVDD) is a musculoskeletal disorder characterized by imbalanced extracellular matrix synthesis and breakdown, consequently contributing to herniated disks, spine instability and spinal stenosis [1, 2]. Chronic lower back pain is considered to result from intervertebral disc degeneration, which brings a heavy economic burden to the society and families [3, 4]. At present, the medical treatment of IVDD is still limited to pain relief and delaying surgery, due to lacking of understanding of underlying pathogenesis $[5,6]$. Therefore, in order to explore novel therapeutic approaches, there is an urgent need to identify the underlying molecular mechanisms of IVDD.

Except using classical experimental approaches to define IVDD, genetic factors have been considered to play emerging roles in the multifactorial etiology of IVDD [7, 8]. Recently, the dysregulated genes 
expression profiles of IVDD have attracted increasing attention of researchers. Among them, MAP2K6 and RHOBTB2 are identified as two potential therapeutic targets of IVDD [9]. Periostin has also been proven to take part in the progression of human IVDD [10]. Hence, using high-throughput technology to screen out IVDD biomarkers in human samples has been shown to be promising to identify more precise criteria of disease diagnostic, classification and prognosis [11-13].

In this study, we identified NDC80, BUB1B and RAD21 genes as hub genes in the pathogenesis of IVDD utilizing the bioinformatics method. Besides, increased expression of NDC80, BUB1B and RAD21 in IVDD rats verified these genes associations with IVDD. Furthermore, we found that NDC80, BUB1B and RAD21 genes silencing could elevate the expression of aggrecan and collagen II, promote cell cycle and proliferation, and inhibit apoptosis of NP cells, which provides insight into the underlying mechanisms and therapeutic targets of IVDD.

\section{Methods}

\section{Ethics statement}

This study was performed with the approval of the Ethics Committee of Tianjin Medical University General Hospital. All animal experiments were performed under strict adherence with the Guide for the Care and Use of Laboratory Animal by International Committees.

\section{Microarray Data Analysis}

Gene expression data sets GSE34095, GSE63492 and GSE45856 were downloaded from Gene Expression Omnibus. For human NP cells in GSE34095, three degenerative samples were collected from IVDD patients and 3 non-degenerative samples were considered to be control group. MicroRNA expression profiling of GSE63492 was obtained from 5 IVDD patients, compared with 5 cadaveric discs. In relation to the miRNAs in GSE45856, three IVDD specimens and 3 traumatic intervertebral disc specimens were individually analyzed using microarray techniques. After data conversation and normalization[14], we performed differential analysis between degeneration samples and control [15]. We set $\mid$ Log (fold change) $\mid>1$ and adjust $P$ value $<0.05$ as the thresholds to screen out DEGs and differentially expressed miRNAs. Then, the target genes of the differentially expressed miRNAs were predicted by combining two online miRNA databases, miTarBase [16] and targetscan (http://www.targetscan.org).

\section{Functional enrichment analysis of DEGs and target genes}

In order to identify the underlying biological functions in IVDD, Gene Ontology (GO) and Kyoto Encyclopedia of Genes and Genomes (KEGG) enrichment analysis was performed on the DEGs using Database for Annotation, Visualization, and Integration Discovery (DAVID) database (https://david.ncifcrf.gov/) [17]. 
The Search Tool for Retrieval of Interacting Genes (STRING) online tool was utilized to construct the PPI of DEGs. A confidence score $>0.4$ was representative of statistical significance. Then, the visualization of PPI network was performed by Cytoscape software.

\section{Animal grouping and modeling}

A total of 25 male Sprague-Dawley (SD) rats ( 3 months old, $180 \pm 20 \mathrm{~g}$ ) were from the medical science experimentation center, SUN YAT-SEN UNIVERSITY (Guangzhou, China). The rats were raised individually and freely to eat and drink under $25^{\circ} \mathrm{C}$ conditions with humidity of $50 \sim 70 \%$ for one month. Next, 15 rats were randomly included in the IVDD group, while the remaining 10 rats were included in the normal group. The rats were anaesthetized by intraperitoneal injection with $3 \%$ pentobarbital sodium $(40 \mathrm{mg} / \mathrm{kg}, \mathrm{P} 3761$, Sigma-Aldrich Chemical Company, St Louis MO, USA). A longitudinal incision was made along the mid line of the rats back. After incision of subcutaneous tissues, the fascia surrounding the intervertebral disc was isolated and the sacrospinalis was then stripped. The spinous process (L1-6) was excised with the interlaminar ligaments cut in order to establish the IVDD rat model. Rats of the normal group were only subject to an incision of the subcutaneous tissue and suture after their operation followed by a return to normal feeding. The rats were injected with $80,000 \mathrm{U} / \mathrm{d}$ penicillin over a period of three days. After 3 months, the rats were euthanized through an intraperitoneal injection of excessive anesthesia for followup experiments.

\section{Assessment of IVDD models}

Three months after the operation, microcomputed tomography (micro-CT) and magnetic resonance imaging (MRI) were used to assess the alterations to disc height and vertebral endplate with degeneration. Three rats chosen randomly from both IVDD group and normal group were euthanized through an intraperitoneal injection of excessive anesthesia. Subsequently, micro CT and sagittal T2weighted images were obtained using SkyScan 1172 (SkyScan, Belgium) and 3.0-T MRI scanner (GE, Chicago, USA), respectively. Then, their respective intervertebral discs (L1-6) and adjacent vertebrae were collected. Histological staining including hematoxylin-eosin (HE) and safranin-O/Fast-greening staining was performed for assessment of cell morphology, extent of endplate ossification, and cartilage matrix of the endplate.

\section{Immunohistochemistry}

Three rats were randomly selected from both the IVDD and normal groups. After euthanasia, rat intervertebral disc samples were fixed with 10\% formaldehyde and later cut in a successive manner into 4 $\mu \mathrm{m}$ slice sections. The sections were permitted to react with diluted RAD21 (1: 500, ab154769, Abcam, Inc, MA, USA), RAD21 (1: 100, ab109496, Abcam, Inc, MA, USA), and BUB1B (1: 200, ab54894, Abcam, Inc, MA, USA). As for Collagen II, the climbing flake of NP cells treated for $7 \mathrm{~d}$ were taken, added with diluted Collagen II (1: 500, ab34712, Abcam, Inc, MA, USA). And then uniformly, the sections were added with biotin-labeled goat anti-mice secondary antibody (A21020, Abcam, USA), horseradish peroxidase-labeled 
streptavidin working solution (DF7852, BIOSAMITE, Shanghai, China) and diaminobenzidine (DAB) to be observed under a light microscope.

\section{Real-Time quantitative Polymerase Chain Reaction}

Three rats were randomly selected from both the IVDD and normal groups. Then, total RNA was extracted using the trizol method. RNA was reversely transcribed into cDNA using the Takara reverse transcription kit (RR047, Takara Biotechnology Ltd., Dalian, China). RT-qPCR was performed with instructions of SYBR®Premix Ex TaqTM II kit (Takara Biotechnology Ltd., Dalian, China). Glyceraldehyde-3-phosphate dehydrogenase (GAPDH) was used as the internal reference.

The primer sequences were listed as follows: RAD21: CAGAGTAGAAGAGATAACCATGAG, ATTCCAAAGTCACCGAAGTC; NDC80: TGCTATGAGAAGTTCATGACTG, ATACAAGTCCTTCAGCTTCG; BUB1B: CCATGATAAAGGCATCTTCTCAG, AGTCCTCATTCAGACGCTC; Aggrecan:

GCTACGGAGACAAGGATGAGTTC, CGTAAAAGACCTCACCCTCCAT. GAPDH: AACTCCCATTCTTCCACC, TTGTCATACCAGGAAATGAGC; The 2- $\triangle \mathrm{Ct}$ methods was used to calculate RNA relative quantitative results.

\section{Isolation and cultivation of NP cells}

The NP cells were collected from the remaining IVDD rats intervertebral discs (L1-6) that was digested with $0.25 \%$ trypsin (25200-056, Gibco Company, Grand Island, NY, USA) for $15 \mathrm{~min}$ at $37^{\circ} \mathrm{C}$. The collected cells were then seeded into a $25 \mathrm{~mm}^{2}$ culture flask with a density of $2 \times 10^{4}$ cells $/ \mathrm{mL}$ and cultured in a $5 \%$ $\mathrm{CO}_{2}$ incubator added with dulbecco's minimum essential medium (DMEM) medium (C11330500BT, Invitrogen, Car, Cal, USA) containing $3 \%$ fetal bovine serum (FBS) at $37^{\circ} \mathrm{C}$. The third generation of NP cells were used for the following experiments.

\section{Cell grouping and transfection of Small Interfering RNA (siRNA)}

Based on the total mRNA sequences of NDC80, BUB1B and RAD21 genes (NM_006101, NM_001211 and NM_006265) in Genbank, the RNAi oligonucleotide sequences of NDC80, BUB1B and RAD21 genes (three pairs of sequences per siRNA) and the negative control (NC) sequences were designed and synthesized by Guangzhou RiboBio Co., Ltd. (Guangdong, China). The NP cells were subsequently assigned into the blank group (no transfection), NC group (transfected with empty vector), siRNA-NDC80 group (transfected with siRNA-NDC80-1/2/3), siRNA-BUB1B group (transfected with siRNA-BUB1B-1/2/3) and siRNA-RAD21 group (transfected with siRNA-RAD21-1/2/3). The siRNA with the highest transfection efficiency was selected from the 3 pairs of siRNA-NDC80, siRNA-BUB1B and siRNA-RAD21. The primer sequences for siRNA-NDC80, siRNA-BUB1B, siRNA-RAD21 are listed as follows $₫$ siRNA-NDC80-1》 GCATATGAACTAGCTACTT; sIRNA-NDC80-2: GCTCTCTCCTTCTGCCTTA;

siRNA-NDC80-3: GCATCTACTCAGCCTCTAA; siRNA-BUB1B-1 GCAATGAGCCTTTGGATAT; siRNA-BUB1B-2: GCTGAAGAGTACGAAGCTA; siRNA-BUB1B-3: CCAGTGTGCTTCCCAACTT; siRNA-RAD21-1: 
The NP cells were then implanted into a 6 -well plate at a density of $2 \times 10^{5}$ cells/well with each well added with $1.3 \mathrm{~mL}$ serum-free Opti-MEM (31985-070, Gibco Company, Grand Island, NY, USA). Next, $5 \mu \mathrm{L}$ lipofectamine 2000 (Invitrogen Inc., Carlsbad, CA, USA) with $3 \mu \mathrm{g}$ of siRNA expressing plasmid were dissolved with $100 \mu \mathrm{L}$ serum free Opti-MEM culture medium respectively, mixed and permitted to stand at $37^{\circ} \mathrm{C}$ for $5 \mathrm{~min}$. After that, products were added into the corresponding wells with each well containing $200 \mu \mathrm{L}$ corresponding products. Then, the products were placed in incubator for $5 \mathrm{~h}$, and exchanged with a fresh complete medium. Finally, the cells were collected after 48-h of transfection.

\section{3-(4,5-Dimethylthiazol-2-yl)-2,5-diphenyltetrazolium bromide (MTT) assay}

Cells at the logarithmic growth phase and in a well-grown state were seeded into a 96-well culture plate at a volume of $100 \mu \mathrm{L}$ per well with $1 \times 10^{4}$ cells per well. After cell adherence for $24 \mathrm{~h}$, they were placed into a $5 \% \mathrm{CO}_{2}$ incubator at $37^{\circ} \mathrm{C}$ for further $24 \mathrm{~h}, 48 \mathrm{~h}$ and $72 \mathrm{~h}$ incubation. Later, $20 \mu \mathrm{L}$ MTT solution (5 $\mathrm{mg} / \mathrm{mL}$ ) was added into each well for incubation purposes at $37^{\circ} \mathrm{C}$ for $4 \mathrm{~h}$. After removal of the supernatant, each well was added with $100 \mu \mathrm{L}$ dimethylsulfoxide (DMSO) and oscillated for $10 \mathrm{~min}$. The OD value of each well at $490 \mathrm{~nm}$ was used to drawn the growth curve.

\section{Flow cytometry}

After $48 \mathrm{~h}$ of transfection, the culture medium was discarded and the cells concentration was adjusted to $1 \times 10^{6}$ cells/mL. The cells stained with propidium iodide (PI) containing RNase (GR1-25, SBS Genetech, Beijing, China) were detected at $488 \mathrm{~nm}$ using a flow cytometer (FACS Calibur, Becton, Dickinson and Company, New Jersey, USA) to cell cycle. The transfected cells at the logarithmic growth phase were used to detect cell apoptosis. According to the instructions of Annexin-V-FITC/PI apoptosis kit (KA3805, Abnova, Walnut, CA, USA), flow cytometry at $488 \mathrm{~nm}$ using the $515 \mathrm{~nm}$ and $560 \mathrm{~nm}$ band pass filter to examine the FITC fluorescence and PI fluorescence, respectively.

\section{Statistical analysis}

One-way analysis of variance (ANOVA) was used for comparisons among multiple groups with SPSS 21.0 software. Data were expressed as mean \pm standard deviation, and $p<0.05$ was regarded as statistically significant.

\section{Results}

\section{RAD21, NDC80 and BUB1B were identified as DEGs}

Initially, bioinformatic analysis was applied to identify the differentially expressed genes (DEGs) of IVDD. In regard to GSE34095, a total of 153 DEGs were screened out, including 111 up-regulated genes and 42 
down-regulated genes. Among them, the RAD21, NDC80 and BUB1B genes were upregulated (Fig. 1a). In addition, seven up-regulated miRNAs and 8 down-regulated miRNAs were obtained from GSE63492, among which miR-5100 was one of the down-regulated miRNAs (Fig. 1b). Besides, six up-regulated miRNAs and 9 down-regulated miRNAs were obtained from GSE45856. Among them, miR-1246 and miR3908 were detected as down-regulated miRNAs (Fig. 1C). Based on the prediction analysis, the targeted genes of miR-5100, miR-1246 and miR-3908 were confirmed to be RAD21, NDC80 and BUB1B respectively. Therefore, RAD21, NDC80 and BUB1B were significantly upregulated in degenerative NP cell samples.

\section{RAD21, BUB1B and NDC80 were the hub genes related with cell cycle}

To further elucidate the molecular mechanisms of IVDD, we constructed a PPI network of DEGs from NP cells. Furthermore, it was noted that the NDC80, BUB1B, and RAD21 were the hub genes of NP cells within the PPI network (Fig. 1d).

GO enrichment analysis revealed that the RAD21 and BUB1B genes participate in the cell cycle, mitotic cell cycle, cell apoptosis, programmed cell death and cell death processes. The NDC80 gene was determined to be related to cell cycle as well as in the mitotic cell cycle (Table 1). KEGG pathway enrichment analysis revealed that the RAD21 and BUB1B genes were involved in cell cycle-related signaling (Table 2).

Table 1

The top 5 biological processes of DEGs from the PPI network

\begin{tabular}{|c|c|c|c|}
\hline ID & Description & $P$ value & Genes \\
\hline 0007049 & Cell cycle & 0.000814171 & $\begin{array}{l}\text { TXNIP,CKAP2,PSMC6,RAD21,BUB1B,ID4,NDC80, } \\
\text { NUP43, MCM6 }\end{array}$ \\
\hline 0000278 & Mitotic cell cycle & 0.002794736 & PSMC6, RAD21, BUB1B, ID4, NDC80, NUP43 \\
\hline 0006915 & Apoptosis & 0.004716498 & $\begin{array}{l}\text { ITGB3BP,CKAP2,RAD21,RHOT1,BUB1B,GJA1, } \\
\text { PPP1R13L }\end{array}$ \\
\hline 0012501 & $\begin{array}{l}\text { Programmed cell } \\
\text { death }\end{array}$ & 0.005069056 & $\begin{array}{l}\text { ITGB3BP,CKAP2,RAD21,RHOT1,BUB1B,GJA1, } \\
\text { PPP1R13L }\end{array}$ \\
\hline 0008219 & Cell death & 0.010973939 & $\begin{array}{l}\text { ITGB3BP, CKAP2, RAD21, RHOT1, BUB1B, GJA1, } \\
\text { PPP1R13L }\end{array}$ \\
\hline
\end{tabular}


Table 2

The top 5 KEGG pathway of DEGs from the PPI network

\begin{tabular}{|llll|}
\hline ID & Pathway & Pvalue & Genes \\
\hline hsa04110 & Cell cycle & 0.004096934 & RAD21, BUB1B, CDKN1B, MCM6 \\
\hline hsa04974 & Protein digestion and absorption & 0.011756318 & COL9A2, COL9A3, COL3A1 \\
\hline hsa03030 & DNA replication & 0.015820249 & MCM6, RFC4 \\
\hline hsa05162 & Measles & 0.032397308 & FYN, TBK1, CDKN1B \\
\hline hsa04151 & Pl3K-Akt signaling pathway & 0.032726603 & PDGFC,FGFR3,PPP2R3C,CDKN1B \\
\hline $\begin{array}{l}\text { Note: Differentially expressed genes (DEGs);KEGG, Kyoto Encyclopedia of Genes and Genomes; PPI, } \\
\text { protein-protein interaction }\end{array}$ & & \\
\hline
\end{tabular}

\section{Verification Of Ivdd Rats}

To assess whether a successful IVDD rat model was established, the radiologic imaging and histological staining were analyzed. Compared to the normal, the IVDD group of micro-CT scans showed decrease in disc height, increase in osteophyte formation at the vertebral edge. Besides, T2-weighted images showed significant reduction in signal intensity (Fig. 2b). As illustrated in Fig. 2c, IVDD group exhibited a significant intervertebral disc degeneration including a shrunken NP, decreased number and uneven distribution of NP cells, unclear boundary of NP and annulus fibrosis, swelling and fractured inner annulus fibrosis, irregular proliferation and calcification of the cartilage endplate. These findings proved the successful IVDD rat models.

\section{Increased expressions of NDC80, BUB1B and RAD21 in IVDD rats}

RT-qPCR and immunohistochemistry analysis were performed to examine the expression of NDC80, BUB1B and RAD21 in the intervertebral disc tissues of rats (Fig. 3). The results demonstrated that IVDD rats exhibited an elevated mRNA and protein expression of NDC80, BUB1B and RAD21, which was in agreement with the bioinformatics results.

\section{Silencing of NDC80, RAD21 and BUB1B increased the expression of collagen II and aggrecan}

After transfection for $48 \mathrm{~h}$, compared to no matter the blank or NC groups, the siRNA-NDC80-1, siRNABUB1B-2 and siRNA-RAD21-2 groups exhibited the most distinctively down-regulated mRNA expression of NDC80, BUB1B and RAD21, which were used for observing collagen II content (Fig. 4a-C). More importantly, after transfection, the NP cells exhibited a significantly increased content of collagen II aggrecan in the siRNA-NDC80, siRNA-BUB1B and siRNA-RAD21 groups (Fig. 4d-f). These results confirmed the silence efficiency, and showed that silencing of NDC80, BUB1B and RAD21 genes could increase the synthesis of collagen II and aggrecan. 


\section{Silencing NDC80, BUB1B and RAD21 promoted proliferation of NP cells}

As shown in Fig. 4G, compared with the blank group and the NC group, the proliferation rate of the siRNANDC80 group, the siRNA-BUB1B group and the siRNA-RAD21 group was markedly increased at the $48 \mathrm{~h}$ and $72 \mathrm{~h}$ time points (all $p<0.05$ ). The above results demonstrated that silencing of NDC80, BUB1B and RAD21 genes could promote the proliferation of NP cells.

\section{Silencing NDC80, BUB1B and RAD21 accelerated cell cycle and inhibited cell apoptosis}

In terms of the cell cycle distribution and apoptosis rate between the blank and NC groups, no significant difference was found ( $p>0.05$ ). However, the $\mathrm{G} 1 / \mathrm{G} 0$ phase in the siRNA-NDC80, siRNA-BUB1B and siRNA-RAD21 groups were decreased, while the $S$ phase in these gene silencing groups were increased ( $p$ $<0.05)$. There were no obvious changes in the $\mathrm{G} 2 / \mathrm{M}$ phase $(p>0.05)$. Furthermore, after the NDC80, BUB1B and RAD21 gene silencing, the cell apoptosis rate decreased significantly. Collectively, these results suggested that silencing od NDC80, BUB1B and RAD21 genes accelerated cell cycle, which may result in the inhibition of cell apoptosis (Fig. 5).

\section{Discussion}

Although much effort and resources have been invested, the underlying pathogenesis of IVDD still remains poorly understood. Recently, the dysregulated genes expression profiles of IVDD have attracted increasing attention of researchers. To investigate key pathogenic mechanism of IVDD, we identified NDC80, BUB1B and RAD21 as hub genes in the degenerative NP cells utilizing the bioinformatics analysis. Subsequently, increased expressions of NDC80, BUB1B and RAD21 were confirmed in IVDD rats, and silencing NDC80, BUB1B and RAD21 played a protective role in intervertebral disc degeneration by promoting proliferation and inhibiting apoptosis of nucleus pulposus cells.

According to GO enrichment analysis and KEGG analysis, NDC80, BUB1B and RAD21 were predicted to participate in several biological processes and pathways including cell death, apoptosis and cell cycle. The protein encoded by NDC80 confines itself to kinetochore and subsequently acts to mediate the formation of the kinetochore-microtubule structure, which is crucial for the stable kinetochore-microtubule interaction [18-20]. Recent studies have provided evidence highlighting a correlation between the overexpression of NDC80 and the over-activation of the mitotic checkpoint [21]. Mitotic checkpoints could prevent separation errors by adjusting the anaphase time until all of chromosomes have been precisely been attached to the spindle microtubules [22]. In mammal cells, BUB1B gene encodes BubR1 protein, which is also a vital component of mitotic checkpoints that ensures the synchrony of chromosome segregation [23]. Yamamoto et al. found that the up-regulation of BUB1B may be a physiologically compensatory mechanism to the absence of normal checkpoints function [24]. Besides, exogenous BUB1B prevents chromosomal instability in a manner of triggering apoptosis [25]. Moreover, RAD21 is one of the four subunits of cohesion, participating in repairing of DNA double-strand breaks, and growth of mitosis [26]. C-terminal cleavage of RAD21 could form degradation products, translocation of which to the cytoplasm initiates apoptosis and causes amplification of the cell death signal [27-29]. Hence, 
considering the importance of three interrelated genes in chromosomal stability and increased expressions of NDC80, BUB1B and RAD21 in IVDD rats, the dysregulation of NDC80, BUB1B, and RAD21 may contribute to the disruption of mitosis and subsequent apoptosis of NP cells as IVDD progresses.

Furthermore, in vitro transfection results verified that NDC80, BUB1B and RAD21 gene silencing accelerated cell cycle progression and suppressed NP cells apoptosis. Consistent with our findings, previous studies have suggested that inhibiting of BUB1, NDC80 and RAD21 expression could regulate the cell cycle progression and apoptosis [30-33]. Additionally, the loss of extracellular matrix (ECM) such as collagen II and aggrecan has been highlighted as a marker of IVDD [34,35]. We found that silencing NDC80, BUB1B and RAD21 could enhance synthesis of ECM component, ameliorating disc degeneration in a rat model of IVDD.

There are also some limitations in our study, which should be taken into consideration when interpreting the results. First, the underlying mechanisms on how NDC80, BUB1B and RAD21 genes silencing promoted proliferation and inhibited apoptosis of nucleus pulposus cells were not thoroughly identified. Secondly, whether there are interactions between NDC80, BUB1B and RAD21 genes or how they interact with each other are not clear. In the end, the role of NDC80, BUB1B and RAD21 genes in human intervertebral disc degeneration also needs more research.

\section{Conclusion}

In conclusion, our study identifies three hub genes of NDC80, BUB1B and RAD21 via bioinformatics analysis, and indicates that silencing of NDC80, RAD21 and BUB1B genes ameliorates intervertebral disc degeneration by promoting proliferation and inhibiting apoptosis of nucleus pulposus cells. These findings suggest that NDC80, BUB1B and RAD21 may serve as potential therapeutic targets for IVDD.

\section{Abbreviations}

IVDD: Intervertebral disc degeneration; DEGs:differentially expressed genes; NP:nucleus pulposus; GO:Gene Ontology; KEGG:Kyoto Encyclopedia of Genes and Genomes; DAVID:Database for Annotation, Visualization, and Integration Discovery; STRING:Search Tool for Retrieval of Interacting Genes; micro$\mathrm{CT}$ :microcomputed tomography; MRI:magnetic resonance imaging; HE:hematoxylin-eosin; siRNA:small interfering RNA; NC:negative control; RT-qPCR:Reverse transcription quantitative polymerase chain reaction.

\section{Declarations}

\section{Ethics approval and consent to participate}

This study was performed with the approval of the Ethics Committee of Tianjin Medical University General Hospital. All animal experiments were performed under strict adherence with the Guide for the Care and Use of Laboratory Animal by International Committees. 


\section{Consent for publication}

Not applicable.

\section{Availability of data and materials}

The datasets used and/or analysed during the current study are available from the corresponding author on reasonable request.

\section{Competing interests}

The authors declare that they have no competing interests

\section{Funding}

The study was supported by grant projects:

International Cooperation Program of National Natural Science Foundation of China (81620108018) and National Natural Science Foundation of China $(81930070,81802197,81772342)$ provided funds in the experimental design, animal purchase and feeding, and reagent purchasing.

Hebei Provincial Natural Science Foundation (H2019110028) and Tianjin key research and development plan, key projects for science and technology support (19YFZCSY00660, 19JCZDJC36300) provided funds in the data analysis and interpretation, and manuscript polishing.

\section{Authors' contributions}

BZ, YYG, WG and WXL: experimental work, and manuscript writing. PP, CX and JYH: data analysis, experimental work. CS and RWD: manuscript editing. XHK and SQF: research design. All authors read and approved the final manuscript.

\section{Acknowledgements}

Not applicable.

\section{References}

1. Sun D, Liu P, Cheng J, Ma Z, Liu J, Qin T. Correlation between intervertebral disc degeneration, paraspinal muscle atrophy, and lumbar facet joints degeneration in patients with lumbar disc herniation. BMC Musculoskelet Disord. 2017;18(1):167.

2. Wang F, Cai F, Shi R, Wang XH, Wu XT. Aging and age related stresses: a senescence mechanism of intervertebral disc degeneration. Osteoarthritis Cartilage. 2016;24(3):398-408.

3. Munir S, Rade M, Määttä JH, Freidin MB, Williams FMK. Intervertebral Disc Biology: Genetic Basis of Disc Degeneration. Current Molecular Biology Reports. 2018;4(4):143-50. 
4. Tendulkar G, Chen T, Ehnert S, Kaps H-P, Nüssler AK. Intervertebral Disc Nucleus Repair: Hype or Hope? International Journal of Molecular Sciences. 2019;20(15).

5. D R, Q H. Y D, L H, J L, KD L. Intervertebral disc transplantation in the treatment of degenerative spine disease: a preliminary study. Lancet. 2007;369(9566):993-9.

6. Richardson SM. Intervertebral disc degeneration therapies in human. BMC Musculoskelet Disord. 2015;16:1.

7. Kepler CK, Ponnappan RK, Tannoury CA, Risbud MV, Anderson DG. The molecular basis of intervertebral disc degeneration. The spine journal: official journal of the North American Spine Society. 2013;13(3):318-30.

8. CK K, RK P, CA T, MV R. DG A. The molecular basis of intervertebral disc degeneration. The spine journal: official journal of the North American Spine Society. 2013;13(3):318-30.

9. Chen Y, Chen K, Li M, Li C, Ma H, Bai YS, et al. Genes associated with disc degeneration identified using microarray gene expression profiling and bioinformatics analysis. Genetics molecular research: GMR. 2013;12(2):1431-9.

10. Tsai TT, Lai PL, Liao JC, Fu TS, Niu CC, Chen LH, et al. Increased periostin gene expression in degenerative intervertebral disc cells. The spine journal: official journal of the North American Spine Society. 2013;13(3):289-98.

11. He J, Xue R, Li S, Lv J, Zhang Y, Fan L, et al. Identification of the potential molecular targets for human intervertebral disc degeneration based on bioinformatic methods. Int J Mol Med. 2015;36(6):1593-600.

12. C MM, CR C, RM A, PS GPP. S, et al. Age-Correlated Phenotypic Alterations in Cells Isolated From Human Degenerated Intervertebral Discs With Contained Hernias. Spine. 2018;43(5):E274-E84.

13. JW L, AC A, SY T. The high-throughput phenotyping of the viscoelastic behavior of whole mouse intervertebral discs using a novel method of dynamic mechanical testing. Journal of biomechanics. 2015;48(10):2189-94.

14. Fujita A, Sato JR, Rodrigues Lde O, Ferreira CE, Sogayar MC. Evaluating different methods of microarray data normalization. BMC Bioinform. 2006;7:469.

15. Ritchie ME, Phipson B, Wu D, Hu Y, Law CW, Shi W, et al. limma powers differential expression analyses for RNA-sequencing and microarray studies. Nucleic acids research. 2015;43(7):e47.

16. Chou CH, Chang NW, Shrestha S, Hsu SD, Lin YL, Lee WH, et al. miRTarBase 2016: updates to the experimentally validated miRNA-target interactions database. Nucleic acids research. 2016;44(D1):D239-47.

17. Huang da W, Sherman BT, Lempicki RA. Systematic and integrative analysis of large gene lists using DAVID bioinformatics resources. Nature protocols. 2009;4(1):44-57.

18. EA S, TN D. CL A. Tight bending of the Ndc80 complex provides intrinsic regulation of its binding to microtubules. eLife. 2019;8. 
19. RR W, J A-B SCH. The Ndc80/HEC1 complex is a contact point for kinetochore-microtubule attachment. Nat Struct Mol Biol. 2007;14(1):54-9.

20. PJ HItV VAV. M D, A M. Multivalency of NDC80 in the outer kinetochore is essential to track shortening microtubules and generate forces. eLife. 2018;7.

21. MA A, RJ M. D V. Antagonism between the dynein and Ndc80 complexes at kinetochores controls the stability of kinetochore-microtubule attachments during mitosis. J Biol Chem. 2018;293(16):575565.

22. B DF, C W. NE D, J P. The Mitotic Checkpoint Complex Requires an Evolutionary Conserved Cassette to Bind and Inhibit Active APC/C. Molecular cell. 2016;64(6):1144-53.

23. Y Y, A O JA. T M, N F, K K, et al. BUBR1 overexpression predicts disease-specific survival after nephroureterectomy in patients with upper tract urothelial carcinoma. Jpn J Clin Oncol. 2016;46(8):754-61.

24. Yamamoto $Y$, Matsuyama H, Chochi Y, Okuda M, Kawauchi S, Inoue R, et al. Overexpression of BUBR1 is associated with chromosomal instability in bladder cancer. Cancer Genet Cytogenet. 2007;174(1):42-7.

25. F LHXLYC, MS Y. T, Z D, et al. Chromium induces chromosomal instability, which is partly due to deregulation of BubR1 and Emi1, two APC/C inhibitors. Cell cycle (Georgetown. Tex). 2011;10(14):2373-9.

26. S B-R CBCA, MA MW, DL H. S, et al. Cohesin promotes the repair of ionizing radiation-induced DNA double-strand breaks in replicated chromatin. Nucleic acids research. 2010;38(2):477-87.

27. AK P, N Z. Q M, D P. Calpain-1 cleaves Rad21 to promote sister chromatid separation. Molecular cellular biology. 2011;31(21):4335-47.

28. F C, M K. M M, Y B, HX, MJ M, et al. Caspase proteolysis of the cohesin component RAD21 promotes apoptosis. J Biol Chem. 2002;277(19):16775-81.

29. C G-M GF, F LRB. G, V S, A C, et al. CyclinD1 Down-Regulation and Increased Apoptosis Are Common Features of Cohesinopathies. Journal of cellular physiology. 2016;231(3):613-22.

30. LL J, L C, YF JHL, ZL WRJL. B, et al. Effect of NDC80 in human hepatocellular carcinoma. World journal of gastroenterology. 2017;23(20):3675-83.

31. Y ML. W, J T, M H, Q L, F J, et al. MicroRNA-450a-3p represses cell proliferation and regulates embryo development by regulating Bub1 expression in mouse. PloS one. 2012;7(10):e47914.

32. SF M. N G, E C, A L, T J, F R, et al. A siRNA screen identifies RAD21, EIF3H, CHRAC1 and TANC2 as driver genes within the 8q23, 8q24.3 and 17q23 amplicons in breast cancer with effects on cell growth, survival and transformation. Carcinogenesis. 2014;35(3):670-82.

33. AW TDJA. B, JA H. HDAC8 Inhibition Blocks SMC3 Deacetylation and Delays Cell Cycle Progression without Affecting Cohesin-dependent Transcription in MCF7 Cancer Cells. J Biol Chem. 2016;291(24):12761-70. 
34. Latridis JC, Godburn K, Wuertz K, Alini M, Roughley PJ. Region-dependent aggrecan degradation patterns in the rat intervertebral disc are affected by mechanical loading in vivo. Spine. 2011;36(3):203-9.

35. Z YCYWHSJW. Z, J C, et al. Melatonin ameliorates intervertebral disc degeneration via the potential mechanisms of mitophagy induction and apoptosis inhibition. J Cell Mol Med. 2019;23(3):2136-48.

\section{Figures}


A

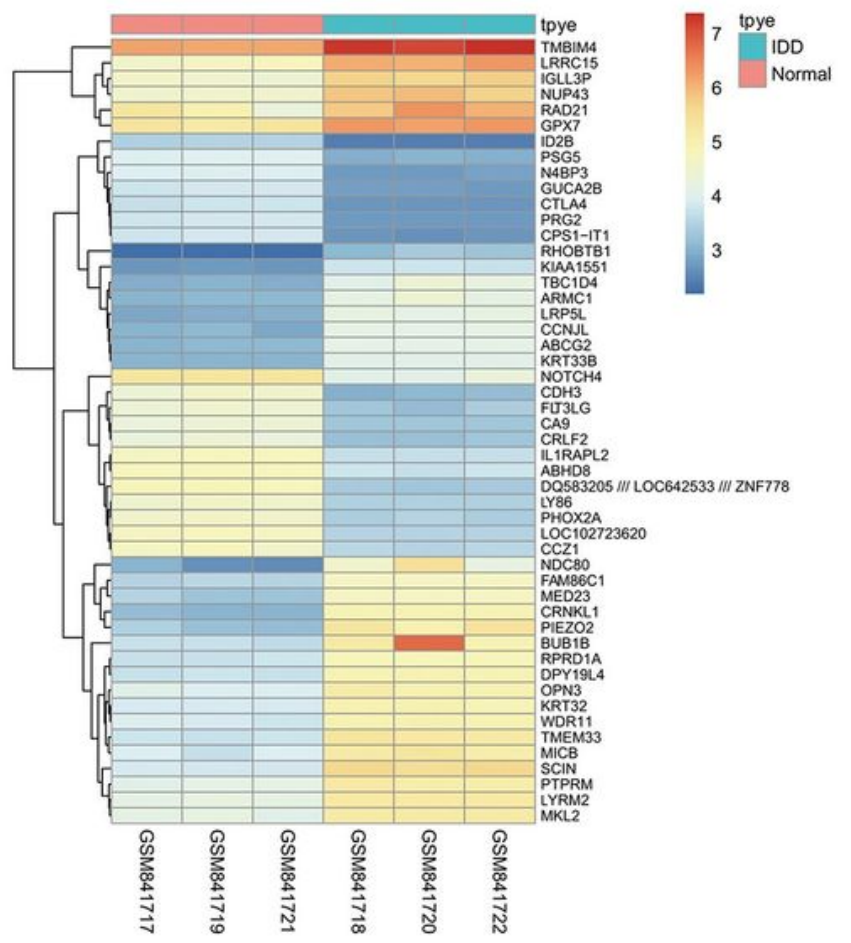

C

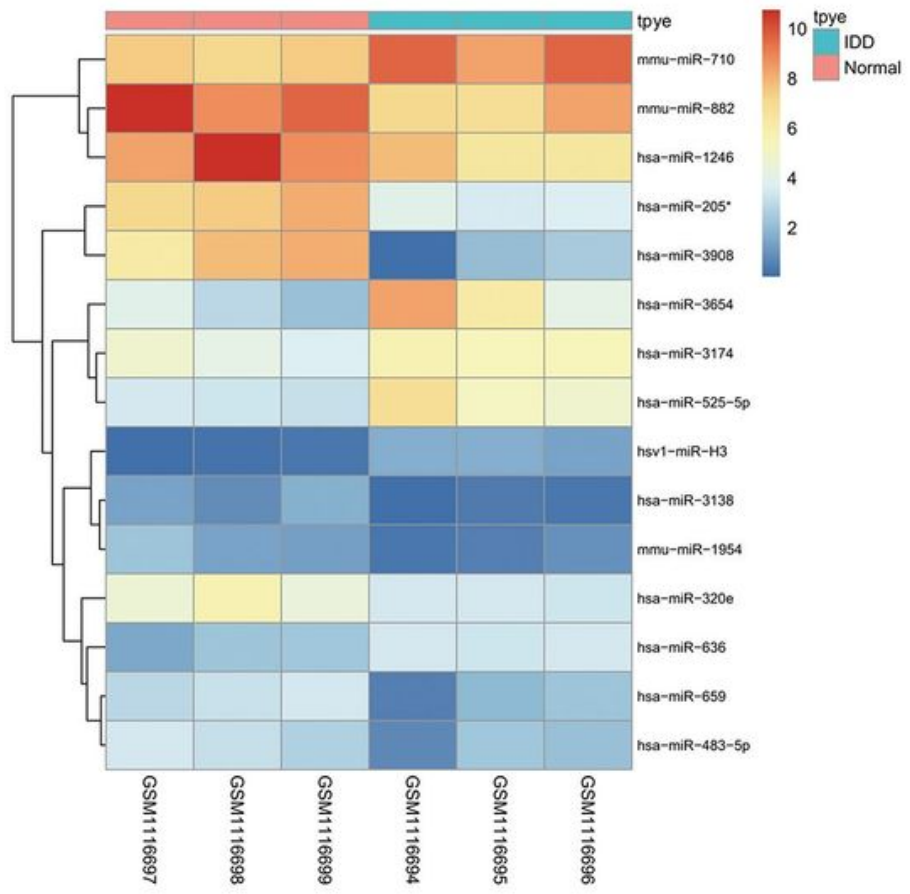

B
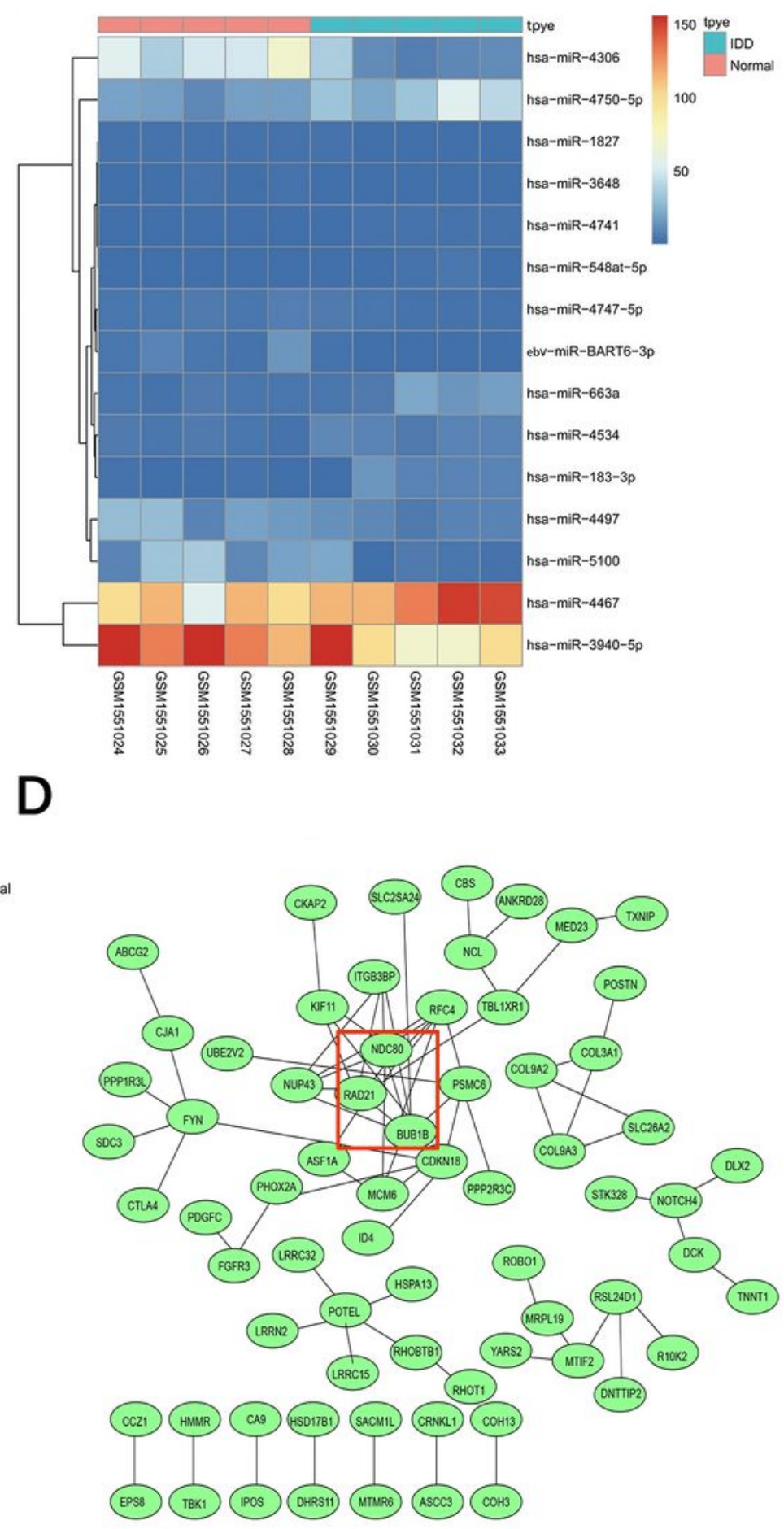

\section{Figure 1}

MiRNA-gene network analysis. a-c, Hierarchical cluster analysis of the significantly upregulated and downregulated genes (A-GSE34095) and miRNAs (B-GSE63492 and C-GSE45856). RAD21, NDC80 and BUB1B were significantly upregulated in degenerative NP cell samples. d, RAD21, NDC80 and BUB1B were identified as hub genes in the PPI network. PPI, protein-protein interaction; NP, nucleus pulposus 

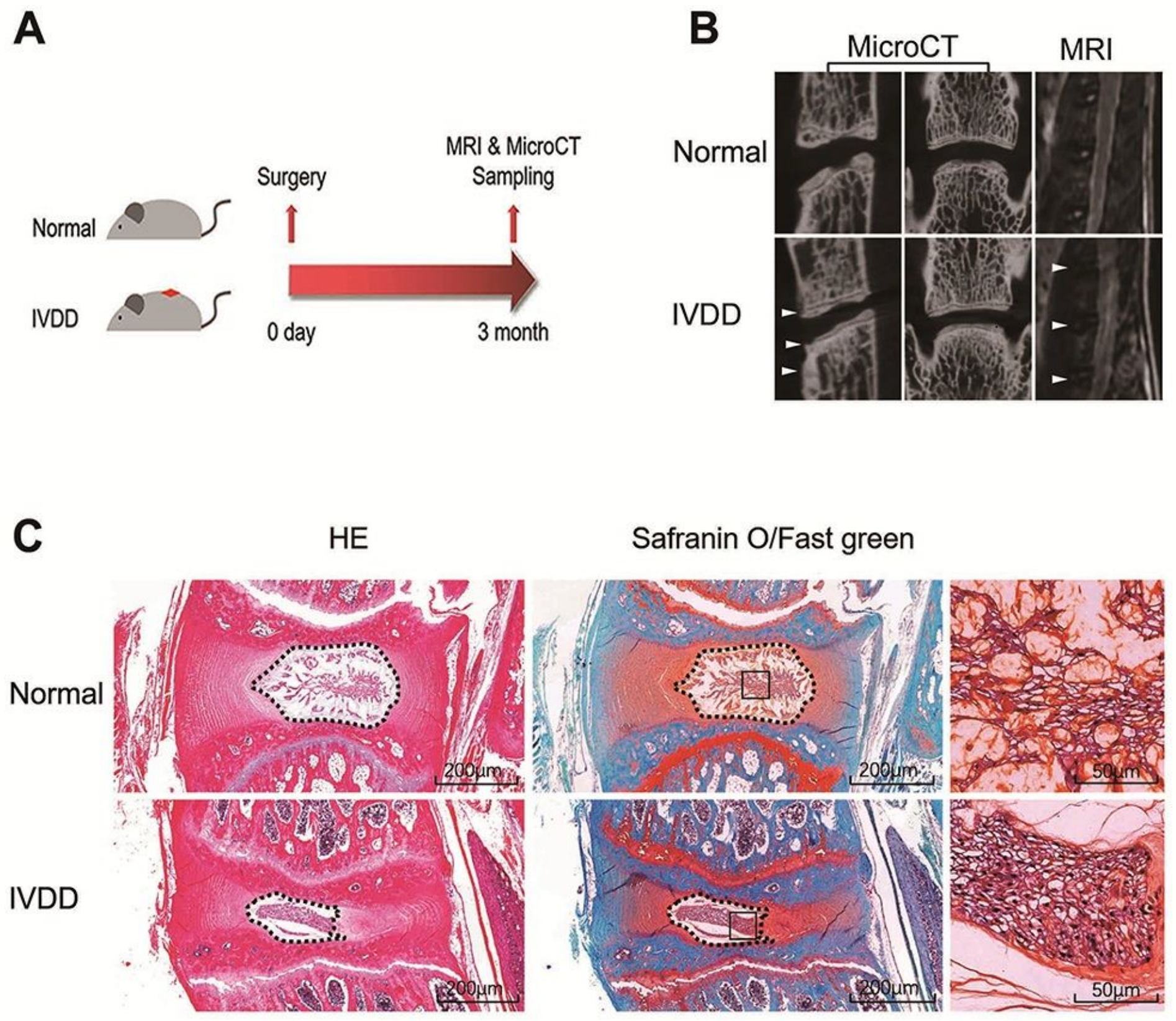

Figure 2

Verification of rat IVDD model by radiologic imaging and histological staining. a, Flow diagram of the experiments in vivo. b, Micro-CT (sagittal and coronal) and MRI (sagittal) were obtained 3 months after surgery. Arrows show the osteosclerosis of vertebrae (bottom left) and significant reduction in signal intensity (bottom right). c, hematoxylin-eosin and safranin-0/fast green staining 
B

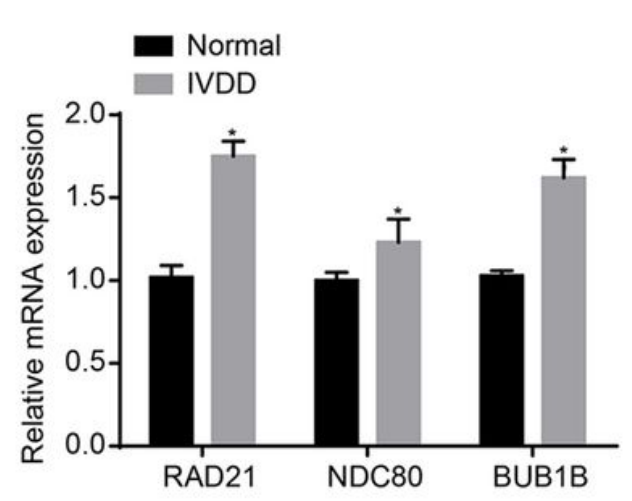

RAD21

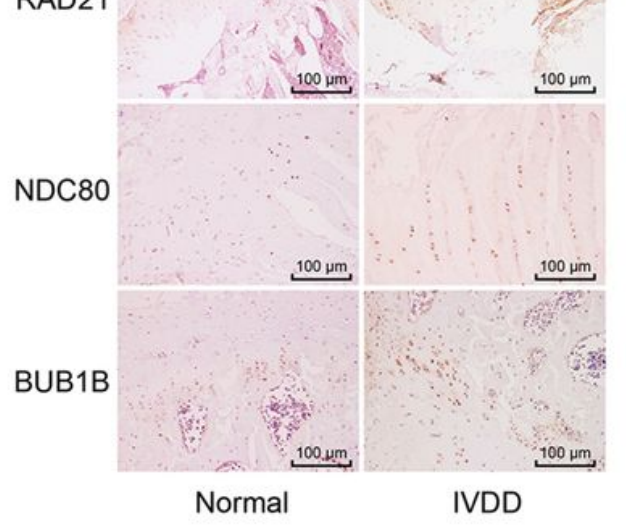

C

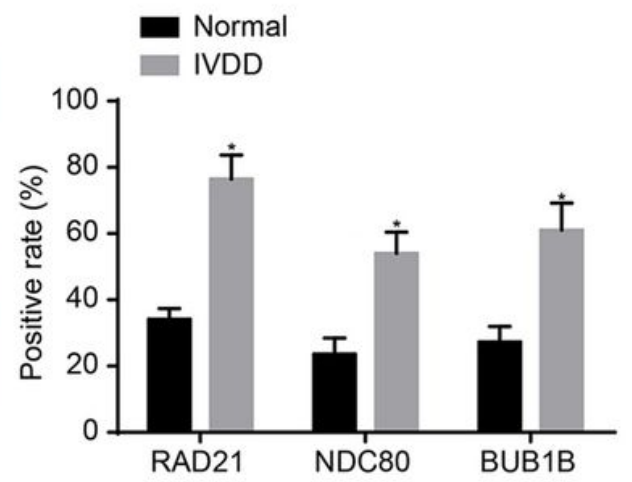

Figure 3

Expression of RAD21, NDC80 and BUB1B were upregulated in rat IVDD model. a, MRNA expression of RAD21, NDC80 and BUB1B examined by RT-qPCR. b-c, Immunohistochemistry analysis of protein expression of RAD21, NDC80 and BUB1B. ${ }^{*} p<0.05$ compared with the normal group 
A

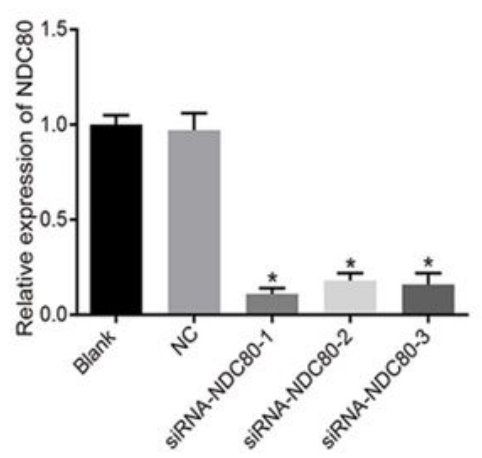

D

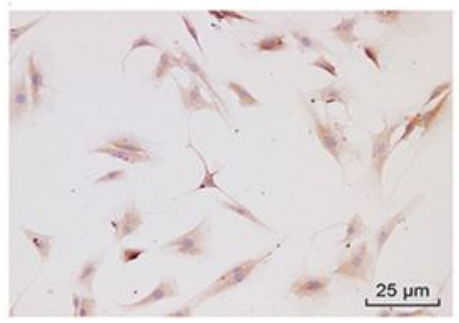

Blank

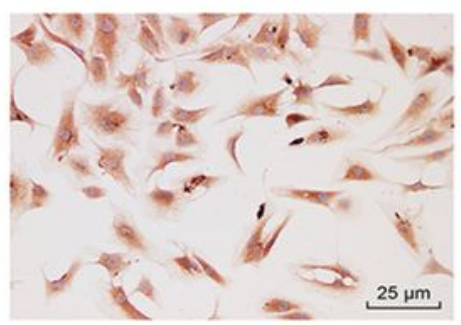

SIRNA-NDC80

F

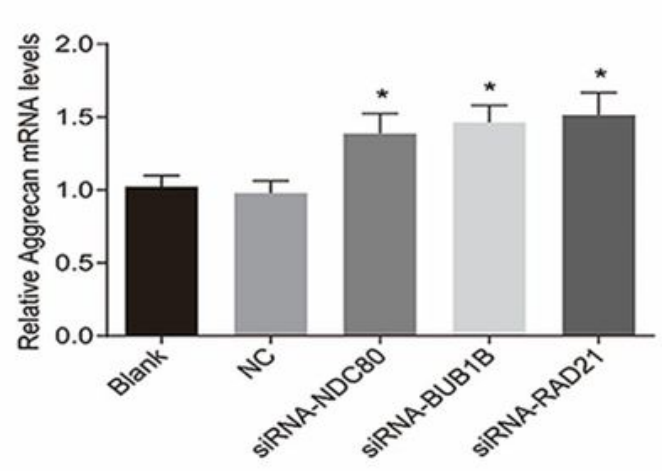

C
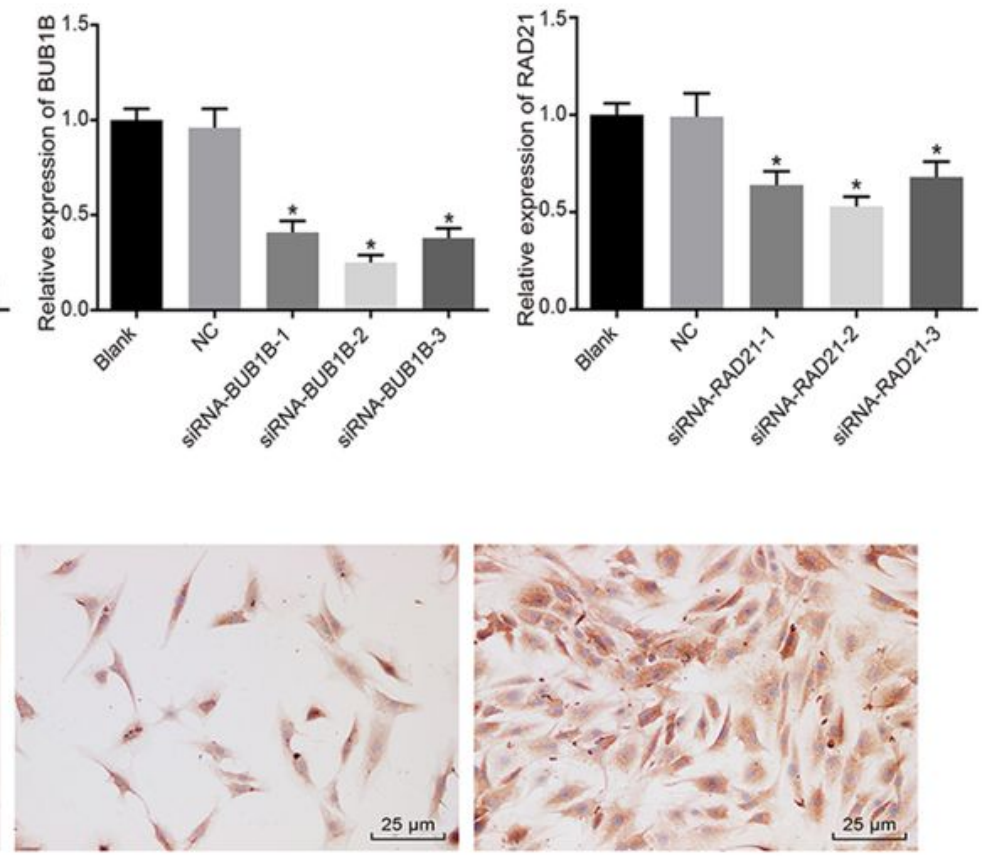

NC

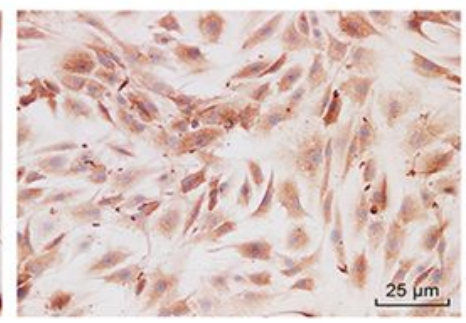

siRNA-RAD21

E

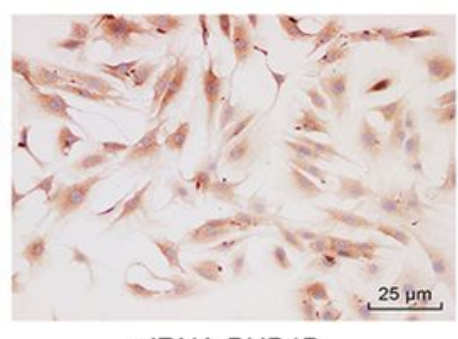

siRNA-BUB1B

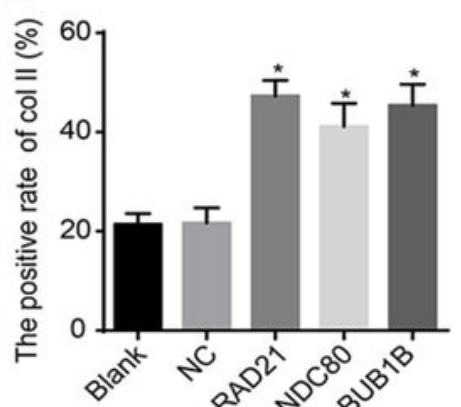

G

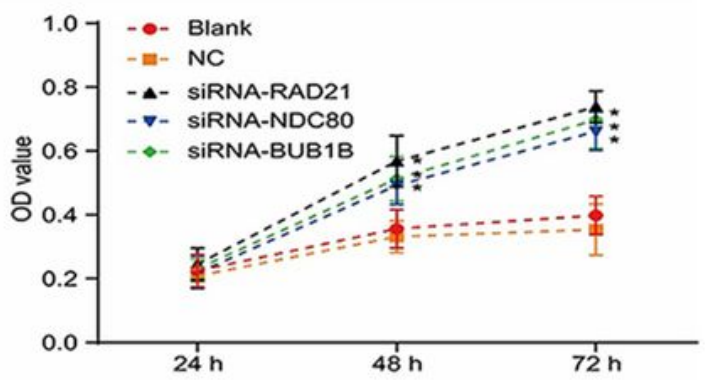

\section{Figure 4}

The effect of silencing NDC80, BUB1B and RAD21 on the expression of collagen II and aggrecan and proliferation of NP cells. a-c, Silencing efficiency of three pairs of siRNAs in the siRNA-RAD21, siRNANDC80, and siRNA-BUB1B group. d-e, Silencing NDC80, BUB1B and RAD21 increased the expression of collagen II of NP cells, determined by immunohistochemistry $(n=6)$. f, Silencing NDC80, BUB1B and RAD21 increased aggrecan content of NP cells, determined by RT-qPCR $(n=6)$. g, Silencing NDC80, 
BUB1B and RAD21enhanced proliferation of NP cells, determined by MTT assay. ${ }^{*} p<0.05$ compared with the blank and NC groups. NC, negative control; NP, nucleus pulposus
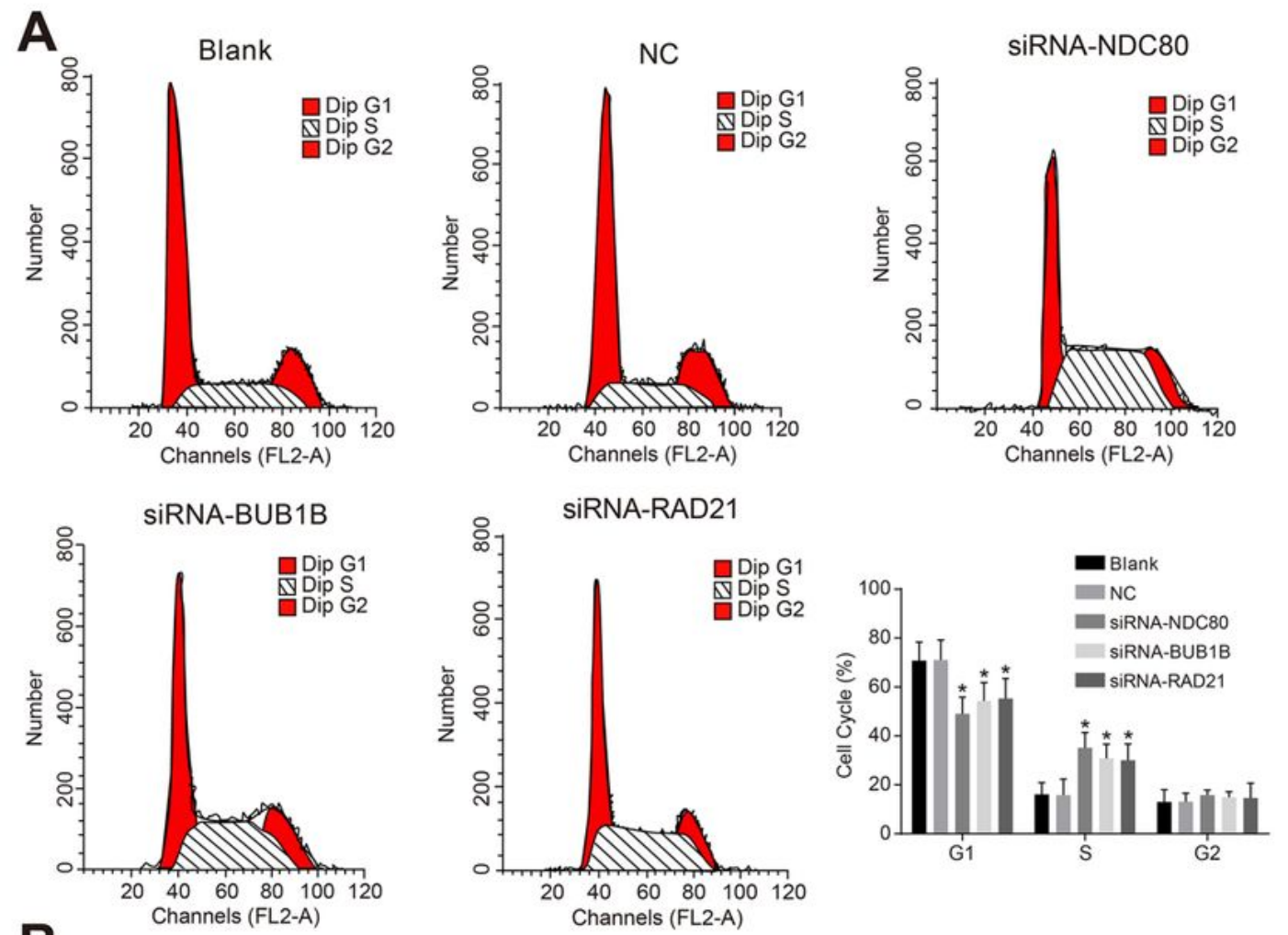

B
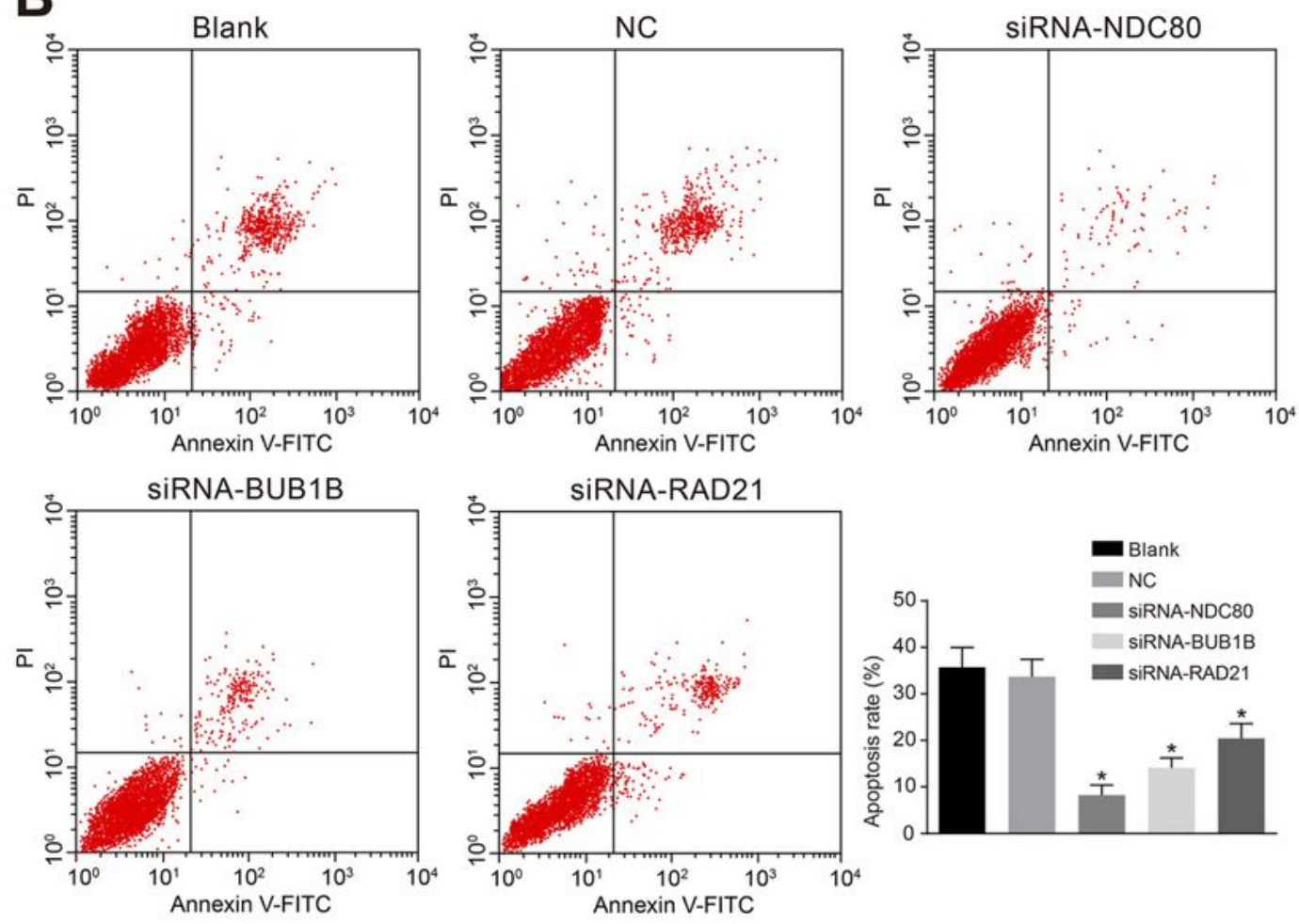

Figure 5

Silencing NDC80, BUB1B and RAD21 accelerated cell cycle and inhibited cell apoptosis of NP cells. a, cell cycle distribution in the blank, NC, siRNA-NDC80, siRNA-BUB1B and siRNA-RAD21 groups, detected by flow cytometry. b, cell apoptosis in the blank, NC, siRNA-NDC80, siRNA-BUB1B and siRNA-RAD21 groups, 
detected by flow cytometry. ${ }^{*} \mathrm{p}<0.05$ compared with the blank and NC groups. NC, negative control; NP, nucleus pulposus

\section{Supplementary Files}

This is a list of supplementary files associated with this preprint. Click to download.

- TheARRIVEGuidelinesChecklist.pdf 